\title{
Expression of transcriptional factor EB (TFEB) in differentiating spermatogonia potentially promotes cell migration in mouse seminiferous epithelium
}

Yue Liu $u^{1,2^{*}}$ D, Yanqin $\mathrm{Hu}^{1,2}$, Li Wang ${ }^{1,2}$ and Chen $\mathrm{Xu}^{1,2^{*}}$

\begin{abstract}
Background: Spermatogenesis is a complex process involving the self-renewal and differentiation of spermatogonia into mature spermatids in the seminiferous tubules. During spermatogenesis, germ cells migrate from the basement membrane to cross the blood-testis barrier (BTB) and finally reach the luminal side of the seminiferous epithelium. However, the mechanism for regulating the migration of germ cells remains unclear. In this study, we focused on the expression and function of transcriptional factor EB (TFEB), a master regulator of lysosomal biogenesis, autophagy and endocytosis, in spermatogenesis.
\end{abstract}

Methods: The expression pattern of the TFEB in mouse testes were investigated by Western blotting and immunohistochemistry analyses. Either undifferentiated spermatogonia or differentiating spermatogonia were isolated from testes using magnetic-activated cell sorting based on specific cell surface markers. Differentiation of spermatogonia was induced with $100 \mathrm{nM}$ retinoic acid (RA). shRNA was used to knock down TFEB in cells. TFEB expression was detected by immunofluorescence, qRT-PCR, and Western blotting. Cell migration was determined by both transwell migration assay and wound healing assay applied to a cell line of immortalized spermatogonia, GC-1 cells.

Results: During testicular development, TFEB expression was rapidly increased in the testes at the period of 7 days postpartum (dpp) to $14 \mathrm{dpp}$, whereas in adult testis, it was predominantly localized in the nucleus of spermatogonia at stages VI to VIII of the seminiferous epithelial cycle. Accordingly, TFEB was observed to be mainly expressed in differentiating spermatogonia and was activated for nuclear translocation by RA treatment. Moreover, knockdown of TFEB expression by RNAi did not affect spermatogonial differentiation, but significantly reduced cell migration in GC-1 cells.

Conclusion: These findings imply that regionally distinct expression and activation of TFEB was strongly associated with RA signaling, and therefore may promote cell migration across the BTB and transport along the seminiferous epithelium.

Keywords: Spermatogenesis, TFEB, Spermatogonia, Cell migration

\section{Background}

Spermatogenesis is a complex process that takes place in the testis and depends on the cooperation between somatic Sertoli cells and developing germ cells across the seminiferous epithelium. In the seminiferous epithelium, Sertoli cells and germ cells are arranged in a highly polarized fashion to efficiently support spermatogenesis

\footnotetext{
* Correspondence: liuyue@shsmu.edu.cn; chenx@shsmu.edu.cn

'Department of Histology, Embryology, Genetics and Developmental Biology, Shanghai Jiao Tong University School of Medicine, Shanghai 200025, China Full list of author information is available at the end of the article
}

by establishing an adequate environment for the production of sperm as well as providing a means of transporting the developing germ cells [1]. During spermatogenesis, the type A spermatogonia undergo mitotic cell divisions to generate type A1 to A4 cells, and some of the type A4 cells differentiate into intermediate spermatogonia, which then divide once to form type B spermatogonia. Then, tetraploid primary spermatocytes are generated from the type B cells and subsequently undergo meiosis to produce diploid secondary spermatocytes and haploid spermatids $[2,3]$. Any alteration of these events, which are tightly

(C) The Author(s). 2018 Open Access This article is distributed under the terms of the Creative Commons Attribution 4.0 International License (http://creativecommons.org/licenses/by/4.0/), which permits unrestricted use, distribution, and 
regulated and precisely coordinated, perturbs spermatogenesis and may lead to male infertility.

In coordination with these cell divisions, the various types of germ cells migrate from the basement membrane to the adluminal side of the seminiferous tubules. One of the key migration steps is when the preleptotene spermatocytes (preL), differentiated from type B spermatogonia, migrate across the blood-testis barrier (BTB), specifically at stage VIII of the seminiferous epithelial cycle $[4,5]$. This migration occurs concomitantly with a peak in retinoic acid (RA) levels present at stages VII and VIII of the seminiferous epithelium cycles, and directs the sequential and distinct programs of spermatogonial differentiation and meiotic initiation $[6,7]$. The capacity of RA to control spermatogonial differentiation, meiotic initiation as well as maintenance of the cycle of the seminiferous epithelium has been well studied over the last couple of years, whereas the relationship between RA and cell migration associated with meiotic initiation is less well understood.

Cell migration is a highly coordinated, multi-step process under the tight control of multiple signaling events. Several studies have shown that the microtubule and F-actin based cytoskeletons in Sertoli cells are crucial for the transport of germ cells into the seminiferous epithelium $[4,8,9]$. However, the molecular mechanisms by which germ cells themselves regulate the timely and efficient migration across the BTB remains virtually unknown. Recently, intracellular vesicular trafficking related to either endocytosis or autophagy, coordinated by the endosome, autophagosome and lysosome systems, has been described as regulators of cell migration [10-15]. Numerous autophagy mediated cargoes and endosomal factors have been shown to modulate cell migration, and the pathways that regulate adhesion turnover or endocytic trafficking have emerged as major players in the transport and recycling of the matrix proteins, adhesion proteins and chemotactic receptors involved in the local regulation of each step of cell migration $[11,12,16]$.

A gene network regulating lysosomal biogenesis and function was discovered recently, at the center of which was the master gene regulator, transcription factor $E B$ (TFEB). TFEB recognizes and binds E-box sequences (5'-CANNTG-3') present in most of the lysosomal related genes $[17,18]$. Moreover, TFEB was also found to modulate the expression of genes involved in processes such as lysosomal biogenesis, lysosomal acidification, exocytosis, autophagy and endocytosis [19-21]. Activation and nuclear translocation of TFEB is mainly controlled by its phosphorylation status, which is modulated in response to various stimuli, such as growth factors and nutrients, and depends on the effects of two major players: the mechanistic target of rapamycin complex 1 (mTORC1) and the phosphatase, calcineurin [22-27]. Very recently, enhancement of TFEB expression and activity has been shown in several cancer cells where it helps to increase tumor growth and malignancy through enhancing cancer cell autophagy and upregulating the endosomal-lysosomal compartment [28, 29]. However, the role and regulation of TFEB in spermatogenetic cells in testis, including spermatogonia, has not been investigated.

To address this question, we investigated the expression pattern of TFEB in mouse testis and spermatogonia and used knockdown experiments to test the role of TFEB in migration and differentiation. In the present study, we show for the first time that TFEB is expressed in differentiating spermatogonia and is activated in response to RA signaling, and therefore potentially plays a role in promoting of cell migration.

\section{Methods}

\section{Animals}

C57BL/6 mice were purchased from the Shanghai Laboratory Animal Center. All of the mice were acclimated in the Animal Center of Shanghai Jiao Tong University School of Medicine. Animal experiments were conducted according to the International Guiding Principles for Biomedical Research Involving Animal, as promulgated by the Society for the Study of Reproduction. This research program was approved by the ethic committee of Shanghai Jiao Tong University School of Medicine (NO. A2015-034).

\section{Histology and immunohistochemistry (IHC)}

Testes were fixed using Bouin's solution and were embedded in paraffin. Specimens were sliced into $5 \mu \mathrm{m}$ thick sections and mounted on glass slides, followed by deparaffinization and rehydration. The sectioned testicular and epididymal tissues were then stained with hematoxylin and eosin (H\&E) and observed under a microscope (Olympus BX53, Tokyo, Japan).

For IHC staining, paraffin sections were dewaxed and rehydrated, followed by antigen retrieval through boiling the tissue for $15 \mathrm{~min}$ in $10 \mathrm{mM}$ citrate buffer, $\mathrm{pH}$ 6.0. Then, the Histostain LAB-SA Detection kits (Invitrogen, MD, USA) were applied according to the manufacturer's instructions. Primary antibody against TFEB (1:100 dilution, Santa Cruz Biotechnology, CA, USA) and the control applied normal IgG were performed overnight at $4{ }^{\circ} \mathrm{C}$. The sections were stained using $\mathrm{DAB}$ and nuclei were counterstained with hematoxylin. Digital images were taken under a microscope (Olympus BX53).

\section{Magnetic-activated cell sorting}

Magnetic-activated cell sorting was used to isolated spermatogonia from testes based on cell specific surface markers modified from a previously described method [30]. Briefly, testicular cells were collected from 7-day-old 
C57BL/6 mouse testes by enzymatic digestion with $1 \mathrm{mg} /$ $\mathrm{ml}$ collagenase IV (Gibico, USA), $2.5 \mathrm{mg} / \mathrm{ml}$ hyaluronidase (Sigma, USA) and $1 \mathrm{mg} / \mathrm{ml}$ DNase I (Sigma), and followed by differential plating in DMEM/F12 medium supplemented with $10 \%$ fetal bovine serum (FBS) (Gibico) for $2 \mathrm{~h}$ at $34{ }^{\circ} \mathrm{C}$ to remove potential contamination of Sertoli and myoid cells. Then, the single cell suspensions were incubated with rat anti-mouse CD90.2 antibody (anti-Thy1, 1: 20 dilution; Miltenyi Biotec, Germany) or rat anti-mouse CD117 antibody (anti-cKit, 1: 20 dilution; Miltenyi Biotec) in $0.1 \mathrm{ml}$ of PBS containing $1 \% \mathrm{FBS}$ at $4^{\circ}$ $\mathrm{C}$ for $20 \mathrm{~min}$. The cells were further sorted on an MS separation column (Miltenyi Biotec) according to the manufacturer's instructions and separated into positive and negative fractions (Additional file 1: Figure S1).

\section{Flow cytometry assay}

Detection of cell purity was carried out by indirect immunofluorescent staining in conjunction with flow cytometry. The immunofluorescent staining and flow cytometry assay were performed as described [31]. The isolated spermatogonia were fixed in $4 \%$ paraformaldehyde for $10 \mathrm{~min}$ at room temperature. Cell samples were labeled with GFRA1 monoclonal antibody (1:200 dilution; Santa Cruz Biotechnology), c-Kit polyclonal antibodies (1:200 dilution; Abcam, USA) and subsequently with fluorescence-labeled secondary antibodies (1:500 dilution; Biotium, USA). Flow cytometry measured the spermatogonia fluorescence signals (Becton Dickinson, Beckman Coulter, Brea, CA, USA). Cell Quest software analyzed the emission originating from at least 10,000 to 20,000 events (Beckman Coulter).

\section{Sertoli cell and germ cell preparation}

Sertoli cell isolation was carried out as described [32, 33] with some modifications. Briefly, testes of adult mice were decapsulated in HBSS containing collagenase $(1 \mathrm{mg} / \mathrm{ml}$ ) for $20 \mathrm{~min}$ and seminiferous tubules were collected by sedimentation. Then, seminiferous tubules were dispersed in a HBSS solution containing collagenase $(1 \mathrm{mg} / \mathrm{ml}) /$ hyaluronidase $(1 \mathrm{mg} / \mathrm{ml}) / \mathrm{DNase}$ $(0.4 \mathrm{mg} / \mathrm{ml})$ for $20 \mathrm{~min}$ at $34{ }^{\circ} \mathrm{C}$. After washing with PBS, an additional digestion step was performed with accutase cell dissociation reagent (Innovative Cell Technologies, USA) for $15 \mathrm{~min}$ at $34{ }^{\circ} \mathrm{C}$. The tubular pellet was washed with PBS and Sertoli cells were freed from the seminiferous epithelium by resuspending the pellet in DMEM/F12 medium containing 5\% FBS. Myoid cells were removed by differential adhesion at the first $30 \mathrm{~min}$ and Sertoli cells were further cultured overnight at $34{ }^{\circ} \mathrm{C}$. The main germ cell fraction in the supernatant was pelleted by centrifugation at $300 \mathrm{~g}$. During Sertoli cell culture, contaminating germ cells were removed by hypotonic shock.

\section{Analyses of gene expression}

Total RNA was isolated using Trizol (Invitrogen) and first-strand cDNA was synthesized using PrimeScript RT Master Mix (Takara, DaLian, China) for reverse transcription-polymerase chain reaction (RT-PCR). For real-time PCR, SYBR Green PCR Master Mix (Takara) were used in accordance with the manufacturer's protocol (Applied Biosystems, USA). Transcript levels were normalized relative to those of $\beta$-actin. PCR conditions were $95{ }^{\circ} \mathrm{C}$ for $5 \mathrm{~min}$, followed by 40 cycles at $95{ }^{\circ} \mathrm{C}$ for $15 \mathrm{~s}$, and $60{ }^{\circ} \mathrm{C}$ for $43 \mathrm{~s}$. Each PCR was run at least in triplicate. Primers used for PCR are referred to a previous study [34] and listed in Additional file 1: Table S1.

\section{Western blotting analysis}

Mice testes or cultured cells were homogenized in RIPA lysis buffer (Thermo Fisher Scientific, USA) containing protease inhibitor cocktail (Roche, USA) on ice for $30 \mathrm{~min}$. Then centrifuged at $12000 \mathrm{~g}, 10 \mathrm{~min}, 4{ }^{\circ} \mathrm{C}$. The proteins in the supernatant were collected and the protein concentrations were determined by the BCA Protein Assay Kit (Thermo Fisher Scientific).

Protein samples $(20 \mu \mathrm{g})$ were separated by using $8-16 \%$ denaturing polyacrylamide gels, then transferred to polyvinylidene difluoride (PVDF) membranes (Millipore, USA) by using a semi-dry transfer apparatus (Bio-Rad, USA). Membranes were blocked with $5 \%$ bovine serum albumin (BSA) for $1 \mathrm{~h}$ at room temperature and immunoblotting was performed overnight at $4{ }^{\circ} \mathrm{C}$ with the TFEB antibodies (1:5000 dilution; Santa cruz) or $\beta$-actin antibodies (1:4000 dilution; Cell Signaling Technology, USA), followed by incubation with secondary antibody conjugated to HRP (Jackson ImmunoResearch, USA). Signals were generated by enhanced chemiluminescence (Millipore) and detected by luminescent image analyzer (GE imagination LAS 4000, USA).

\section{Immunofluorescence analysis}

Cell slides or smears were prepared and then fixed with $4 \%$ paraformaldehyde for $20 \mathrm{~min}$ at $4{ }^{\circ} \mathrm{C}$. Nonspecific binding sites were blocked with 10\% BSA/PBS for $60 \mathrm{~min}$ at room temperature, followed with $0.1 \%$ TritonX-100 permeable treatment for $10 \mathrm{~min}$. Sections were incubated with the TFEB antibodies (1:200 dilution; Santa cruz), GFRA1 antibody (1:200 dilution; Santa cruz) overnight at $4{ }^{\circ} \mathrm{C}$. Then, fluorescence-labeled secondary antibodies (donkey anti-rabbit Alexa Fluor 488, donkey anti-mouse Alexa Fluor 555, 1:500 dilution; Jackson ImmunoResearch) were used. Nuclei were counterstained with DAPI (Sigma-Aldrich). The fluorescence signals were detected under a laser scanning confocal microscope (Carl Zeiss LSM-510, Germany) equipped with an argon laser $(488 \mathrm{~nm})$, a He/Ne laser (543 nm), an EC Plan-NEOFLUAR $63 \times / 1.25$ objective and a LD 
LCI Plan-APOCHROMAT 25×/0.8 objective (Zeiss). Digital images were taken and processed using Aim software (Zeiss Systems).

\section{Primary cell culture of spermatogonia}

Thy1 positive spermatogonia were seeded in 6-cm dishes coated with laminin (BD Biosciences, USA) at a concentration of 5000 cells/well in $\alpha$-MEM medium (Gibico) supplemented with $1 \%$ FBS, $1 \times$ non-essential amino acids (NEAA) (Gibico), $1 \times \mathrm{N} 2-1$ (Gibico), $4 \mathrm{ng} / \mathrm{ml}$ glial cell-derived neurotrophic factor (GDNF) (ProteinTech, USA) and $1000 \mathrm{U} / \mathrm{ml} \mathrm{LIF} \mathrm{(ProteinTech)} \mathrm{[30,} \mathrm{35].} \mathrm{All} \mathrm{cul-}$ tures were maintained at $34{ }^{\circ} \mathrm{C}$ in a humidified $5 \% \mathrm{CO}_{2}$ incubator. In RA inducing differentiation experiment, $100 \mathrm{nM}$ all-trans RA (Sigma) was added to the culture medium for the indicated periods of time to allow spermatogonial differentiation.

\section{RNA interference by lentivirus transduction}

The TFEB shRNA targeting to 5'-GCAGCAGGC TGTCATGCATTA-3' was produced and cloned into the pLenti X1 Puro lentiviral vector (pLenti X1 Puro-shRNA-eGFP-1), which contained an enhanced green fluorescence protein (EGFP) as a reporter gene. Meanwhile, control shRNA lentiviral vector was also constructed. Production of the packaging recombinant viruses were performed by Cyagen (Guangzhou, China). In lentivirus transduction, isolated spermatogonia or GC-1 cells were cultured in six-well plates at a density of $2 \times 10^{5}$ cells per well. Recombinant viruses in culture medium containing $8 \mu \mathrm{g} / \mathrm{mL}$ of polybrene with a muiltiplicity of infection (MOI) of 5 were used for transduction of cells in each well. After infection, the plates were incubated at $34{ }^{\circ} \mathrm{C}$ and the medium was changed $24 \mathrm{~h}$ later. The transduction efficiency was determined by EGFP expression with flow cytometry analysis at $48 \mathrm{~h}$ to $72 \mathrm{~h}$ after transduction. The knock down of TFEB expression was determined using the quantitative PCR method.

\section{Transwell assay}

Cells were cultured with DMEM culture medium containing $10 \%$ fetal bovine serum (FBS) for $24 \mathrm{~h}$ for routine digestion. Cell concentration was adjusted to $1 \times 10^{5} / \mathrm{ml}$ using serum free DMEM culture medium. A cell suspension $(100 \mu \mathrm{l})$ was added to the upper chamber of the transwell plate. The lower chamber of the transwell plate was innoculated with $500 \mu \mathrm{l}$ of DMEM culture medium containing $10 \%$ FBS. Then the tranwell plate was cultured in an incubator at $37{ }^{\circ} \mathrm{C}$ with $5 \% \mathrm{CO}_{2}$. About $24 \mathrm{~h}$ later, the plate was taken out and cotton swab was used to wipe out the cells in the transwell room, followed by PBS wash for three times. Then the transwell was soaked in $95 \%$ ethanol solution for $20 \mathrm{~min}$ to fixation and in crystal violet solution for 5 min, followed by PBS wash for three times. The penetrated cells were counted under a microscope. Each group was set with five transwell rooms, and six view fields were observed for each transwell room. Average value was calculated and obtained. Experiments were conducted for three times to obtain the average value.

\section{Wound healing assay}

The wound healing assay was performed according to a previously described method [36]. Briefly, $4 \times 10^{5}$ cells were cultured in a 6-well plate until they reached a near confluent monolayer. This monolayer was subsequently scratched with a $100 \mu \mathrm{l}$ culture tip. Cellular migration from the two wound fronts was tracked and migration distances recorded after $24 \mathrm{~h}$ using a live imaging system (Nikon, Japan). At least five imaging views were investigated on each plate to quantify the migration rates.

\section{Statistical analysis}

All data were analyzed using SAS 8.2 software, and results are presented as mean \pm SD. Group comparisons were made using Student's t-test where appropriate. One-way analysis of variance (ANOVA) test was used assuming a two-tail hypothesis with $P<0.05$. Differences were considered statistically different when $P<0.05$.

\section{Results}

Expression and localization of TFEB in testis

We used immunohistochemistry and immunoblotting to investigate the expression pattern of TFEB in murine testis and seminiferous epithelium. Immunoblotting analysis indicated that TFEB levels increased during the development of the testis (Fig. 1a). Consistent with this finding, the mRNA levels of TFEB, measured by qRT-PCR, in the developing testis also increased during testis development, with a pronounced increase in expression during $7 \mathrm{dpp}$ to $14 \mathrm{dpp}$ (Fig. 1b), which corresponds to the spermatogonial differentiation and the first instances of meiosis in neonatal testes.

Immunohistochemistry analysis showed that TFEB visibly appeared in spermatogenetic cells in testis after $14 \mathrm{dpp}$ and was mainly expressed in the nucleus of spermatogonia located along the basal region of the seminiferous epithelium as well as in testicular interstitial cells (Fig. 1c). According to the morphological analysis of the seminiferous tubules in adult mice, the immunohistochemistry staining of TFEB in spermatogonia or spermatocytes was mainly present in the seminiferous tubules at stage VI to VIII. At stage VI, TFEB was mainly localized in the nucleus of the differentiating spermatogonia, type B spermatogonia, which displayed an oval-shaped nucleus with more heterochromatin, and were close to or in mitosis into preL. Thus, at stage VII 


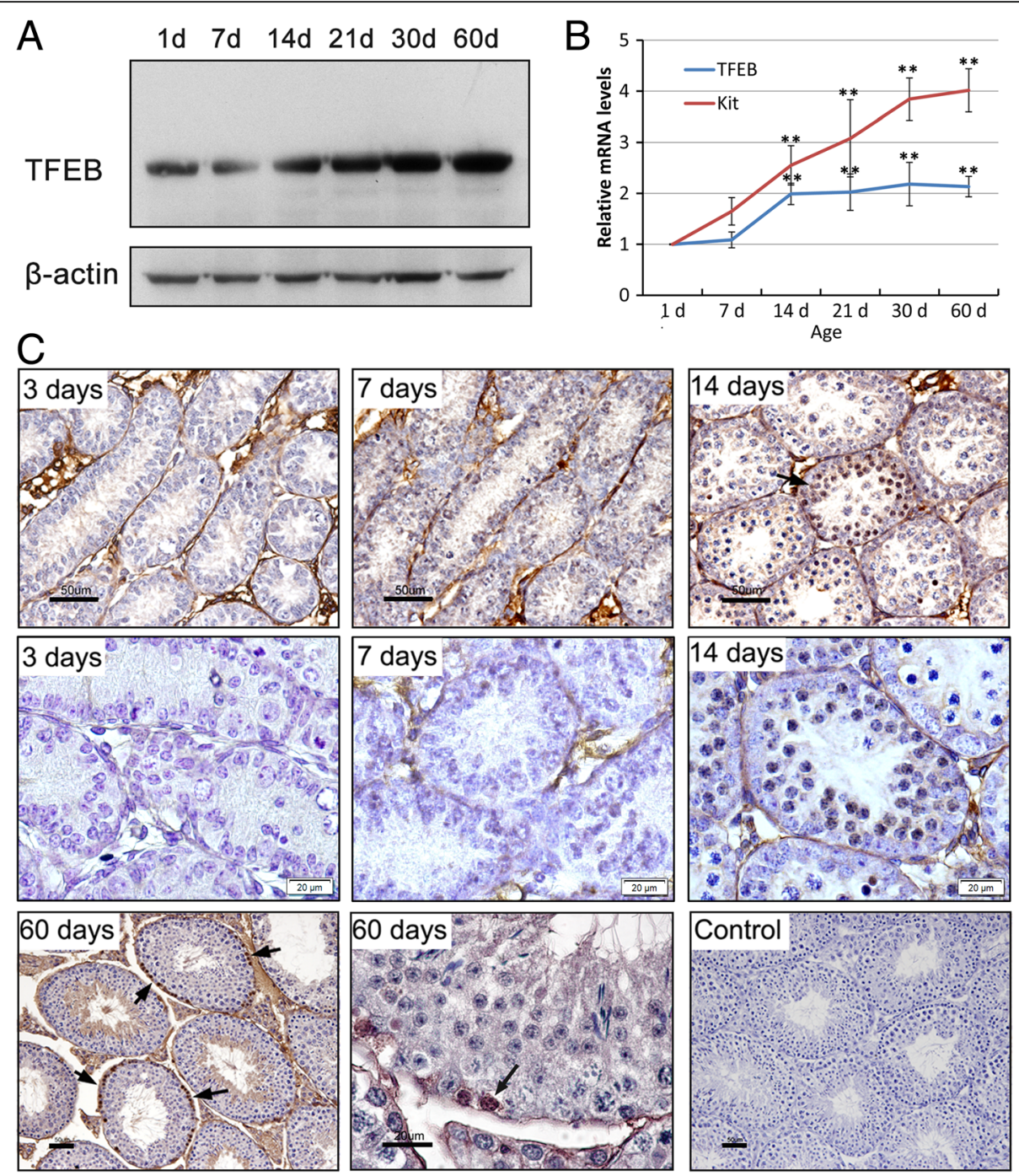

Fig. 1 Expression of TFEB in mouse testes during development. a-b Western blot and quantitative PCR detection of TFEB in 1-, 7-, 14-, 21-, 30and 60-day-old testes, showed a rapid increase in TFEB levels between 7- and 14-day-old testes. Quantitative PCR detection of Kit to confirm the spermatogenetic cell development. Error bars represent SD $(n=5)$. ${ }^{* *} p<0.01$. c Immunohistochemical staining of TFEB in 3-, 7-, 14- and 60-dayold testes sections, showed TFEB expression was little stained in 3- and 7-day testes and significantly positive stained in 14- and 60-day testes. Arrows indicate the staining of TFEB in nucleus. Bar: $50 \mu \mathrm{m}$ or $20 \mu \mathrm{m}$

and VIII, TFEB was mainly localized in the first appeared spermatocytes, the preL (Fig. 2).

\section{TFEB was high expressed in differentiating spermatogonia}

To confirm the expression of TFEB in spermatogonia, both undifferentiated spermatogonia and differentiating spermatogonia were isolated from the $7 \mathrm{dpp}$ testes using magnetic-activated cell sorting. Anti-CD90.2 (Thy1, a cell surface marker for undifferentiated spermatogonia) was applied to isolate the undifferentiated spermatogonia and anti-CD117 (c-Kit, a cell surface marker for differentiating spermatogonia, spermatocyte and spermatid) was applied to isolate the differentiating spermatogonia in $7 \mathrm{dpp}$ testes. The purity of the isolated cells was $87 \pm 6 \%$ and $94 \pm 3 \%$, respectively, verified by flow cytometry analysis (Fig. 3a, b). Cell type-specific marker gene transcripts of the undifferentiated spermatogonia (i.e. Plzf and Gfra1) and differentiating spermatogonia (i.e. Kit and Sohlh2) were identified by qRT-PCR (Fig. 3c). These results indicated that undifferentiated or differentiating spermatogonia were successfully isolated with high purity using magneticactivated cell sorting.

Using qRT-PCR, we found that TFEB mRNA was relatively abundant in the c-Kit positive, differentiating spermatogonia (Fig. 3c). Immunoblotting and immunofluorescence analysis also confirmed high levels of TFEB 


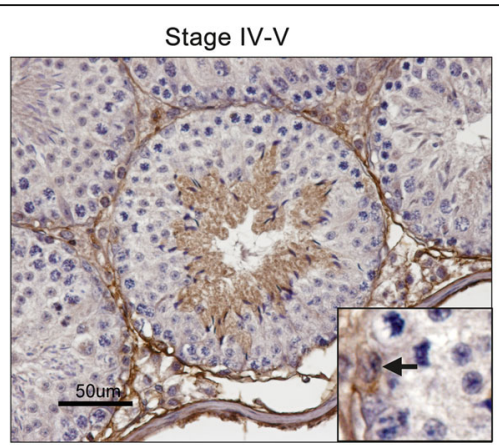

Stage VIII

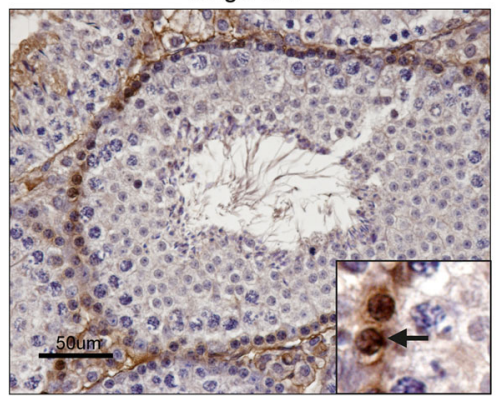

Stage VI

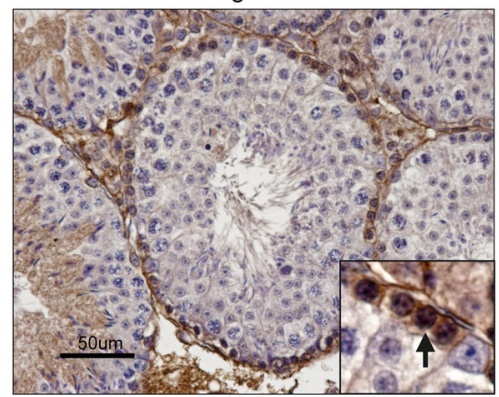

Stage IX

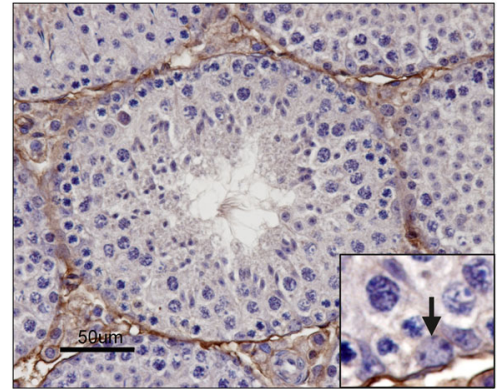

Fig. 2 Localization of TFEB in the seminiferous tubules analyzed by immunohistochemistry staining. TFEB significantly expressed in the basal region of seminiferous tubules between stages VI and VIII of the seminiferous epithelial cycle, and was predominantly localized in the nucleus of type B spermatogonia or preL at stages VI and VIII. The type B spermatogonia displayed an oval-shaped nucleus with some heterochromatin clumps, and the preL also displayed chromatin clumps in nucleus. Arrows indicate the spermatogonia in the basal region of seminiferous tubules. Bar: $50 \mu \mathrm{m}$

protein in c-Kit positive, differentiating spermatogonia (Fig. 3d, e).

\section{Primary culture of undifferentiated spermatogonia and induced spermatogonia differentiation by retinoic acid (RA) treatment}

To simulate spermatogonia differentiation in vitro, the purified Thy1 positive spermatogonia were cultured and then treated with RA to induce cell differentiation. Freshly isolated, Thy1 positive spermatogonia were cultured on laminin coated dishes and consisted of single, paired and aligned cells after being cultured up to 15 days (Fig. 4a). As shown, paired or aligned cells were connected to each other by intercellular bridges (Fig. 4a). Moreover, the cultured cells were identified as undifferentiated spermatogonia by immunofluorescent staining of cell marker, GDNF family receptor alpha 1 (GFRA1) (Additional file 1: Figure S1).

After treatment with RA for 0, 12, 24, 48 and $72 \mathrm{~h}$, the mRNA levels of cell type-specific marker gene transcripts were assessed by qRT-PCR and the results showed that cell markers of the differentiating spermatogonia (i.e. Kit, Sohlh2 and Stra8) were highly expressed after RA treatment for $24 \mathrm{~h}$ (Fig. 4b). Meanwhile, immunofluorescence analysis of GFRA1, a marker of undifferentiated spermatogonia, showed that GFRA1 was localized on the membrane of Thy1 positive spermatogonia and was greatly diminished after RA treatment (Fig. 4c). Cumulatively, these results suggested that Thy1 positive spermatogonia were successfully induced to differentiate with RA treatment.

\section{TFEB activation in response to RA signaling}

To investigate the relationship between spermatogonia differentiation and TFEB activation, the purified Thy1 positive spermatogonia were cultured and then treated with RA to induce cell differentiation. In cultured Thy1 positive spermatogonia, the mRNA level of TFEB was increased about 3-fold after RA treatment for $24 \mathrm{~h}$ (Fig. 4b). More importantly, the ability of TFEB to promote gene transcription is dependent on its nuclear localization, therefore nuclear localization is a marker for the transcription activity of TFEB. Immunofluorescence analysis showed that TFEB localized in the cytoplasm of Thy1 positive, undifferentiated spermatogonia, while it translocated into the nucleus following RA treatment (Fig. 4c). These results suggested that TFEB was activated by RA signaling associated with spermatogonia differentiation.

\section{Reduced expression of TFEB by RNAi did not interfere spermatogonia differentiation induced by RA}

To test whether TFEB was required for RA induced differentiation of spermatagonia, short hairpin RNA (shRNA) expressed by lentiviral vectors (pLenti X1 Puro-shRNAeGFP-1) were used to knockdown the expression of TFEB. 

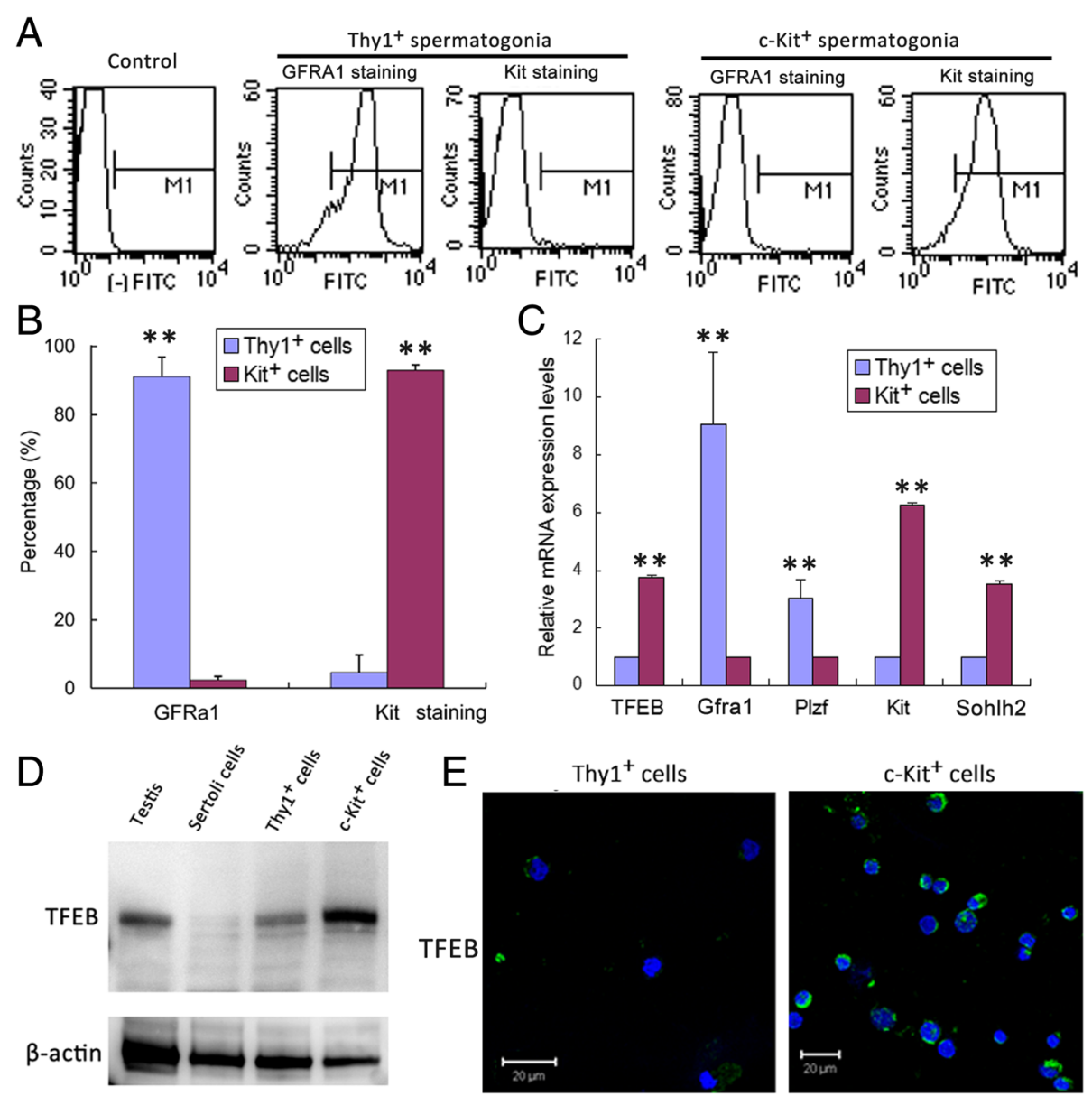

Fig. 3 Identification of spermatogonia isolated from 7-day testes by magnetic-activated cell sorting. $\mathbf{a}$, b The purity of the isolated spermatogonia were identified by flow cytometry analysis, showed that spermatogonia with high purity were isolated by magnetic antibodies. Error bars represent SD $(n=5)$. ${ }^{* *} p<0.01$. c Identification of the isolated spermatogonia by quantitative PCR, showed high levels of Plzf and Gfra1 in Thy1 positive cells and high levels of TFEB, Kit and Soh/h2 in c-Kit positive cells. Error bars represent SD $(n=5) .{ }^{*} p<0.01$. $\mathbf{d}$ Western blot detection of TFEB in testis, Sertoli cells and isolated spermatogonia, indicated it was mainly expressed in c-Kit positive spermatogonia. e Immunofluorescence analysis showed the high expression of TFEB (green fluorescence) in c-Kit positive spermatogonia. Bar: $20 \mu \mathrm{m}$

$72 \mathrm{~h}$ after shRNA lentiviral transduction, TFEB mRNA levels were significantly decreased, by about $73 \%$ compared to untreated cells (blank) or lentiviral vector transducted cells (control). The knockdown efficiency was not reversed after RA treatment $(n=6$, Fig. 4d). Moreover, knockdown of TFEB resulted in significantly increased mRNA levels of spermatogonia differentiation markers (i.e. Kit, Sohlh2 and Stra8), which were similar to those blank or control cells treated with RA (Fig. 4d). Therefore, these results suggest that TFEB does not directly regulate spermatogonia differentiation but might participate in further spermatogenesis through promoting lysosome biogenesis or endocytosis as it does in somatic cells.

\section{Reduced expression of TFEB in GC-1 cells interfere cell migration}

Notably, the morphological analysis of TFEB localization, which showed TFEB is highly expressed in type $B$ spermatogonia in the seminiferous tubules from stage VI to VIII, indicated that TFEB might be involved in the preL migrating across the BTB in the seminiferous tubules at stage VIII.

To identify the effect of TFEB on cell migration, both transwell migration assays and wound healing assays were applied to GC-1 cells, a cell line of immortalized spermatogonia. The lentiviral infection efficiency of GC-1 cells was determined with using an EGFP fluorescent marker by flow cytometer analysis. $48 \mathrm{~h}$ and $72 \mathrm{~h}$ after infection, the EGFP positive cell rate was estimated at $94 \%$ and $9 \%$, respectively $(n=4$, Fig. $5 a)$. TFEB mRNA levels were significantly knocked down at $48 \mathrm{~h}$ and $72 \mathrm{~h}$ after infection, as compared to mock-infected controls and the efficiency of TFEB knockdown was $65 \%$ and $60 \%$, respectively ( $n=6$, Fig. $5 \mathrm{~b}$ ). Moreover, cell survival assays, performed with the MTS method, indicated that knockdown of TFEB had no significant effect on cell 


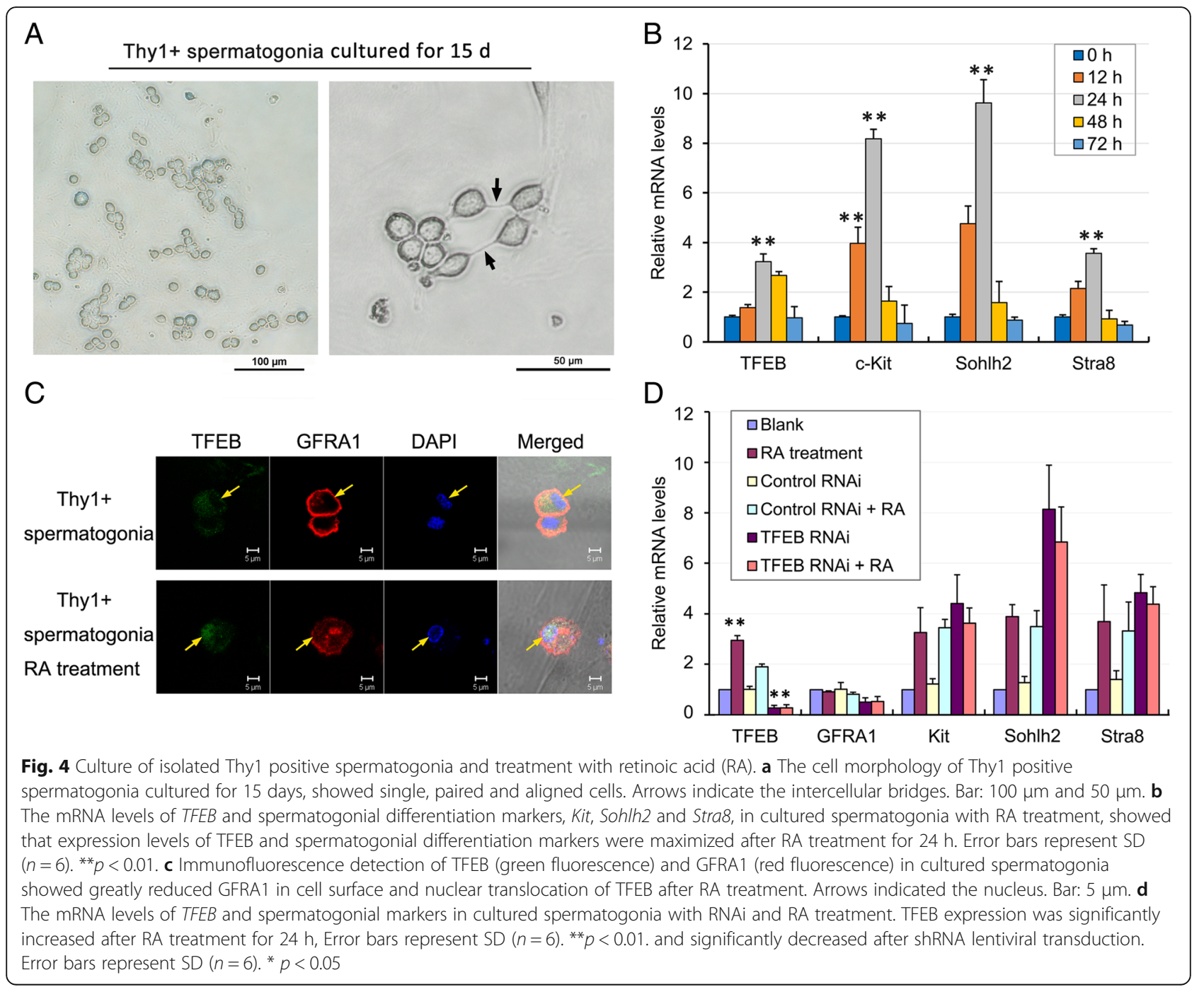

viability at $48 \mathrm{~h}$ and $72 \mathrm{~h}$ after infection $(n=6$, Fig. $5 \mathrm{c})$, suggesting that knock down of TFEB in GC-1 cells was appropriate for further cell migration experiments between $48 \mathrm{~h}$ and $72 \mathrm{~h}$ after lentiviral infection.

In transwell assay, after incubation for $24 \mathrm{~h}$, we counted the number of cells that had penetrated through the transwell (Fig. 6a). The number of migrated TFEB knockdown cells $\left(109 \pm 30 / \mathrm{mm}^{2}\right)$ was significantly lower than that of the blank or control cells (Blank, $211 \pm 19 / \mathrm{mm}^{2}$; Control, $204 \pm 17 / \mathrm{mm}^{2} . n$ $=5, P<0.01$. Fig. $6 \mathrm{~b})$, suggesting that the migration ability in TFEB knockdown group was greatly suppressed. In the wound healing assay, knockdown of TFEB resulted in slower cell migration after $24 \mathrm{~h}$ of incubation (Fig. 6c), as the distance of migration was significantly smaller in cells with TFEB knock down compared with those in blank and control (Blank, 664 $\pm 51 \mu \mathrm{m}$; Control, 659 $\pm 68 \mu \mathrm{m}$; TFEB RNAi, $368 \pm 61 \mu \mathrm{m} . n=5, P<0.01$. Fig. 6d). Cumulatively, these results indicated that the down-regulation of TFEB exerted a repressive effect on cell migration.

\section{Discussion}

The present study has clearly demonstrated the expression of TFEB in mouse testicular development, especially ranging from day 14 to the adult stage. Further we show the specific localization of TFEB in the nucleus of the type B spermatogonia and preL in stage VI to VIII of mouse testis. Expression of TFEB was observed in isolated c-Kit positive, differentiating spermatogonia and its nuclear translocation was associated with cell differentiation induced by RA signaling. Furthermore, knockdown of TFEB in immortalized spermatogonia (GC-1 cells) can inhibit cell migration. These observations, together with the reported master regulating effect of TFEB on lysosome function and autophagy, suggest its important role in germ cell migration. 

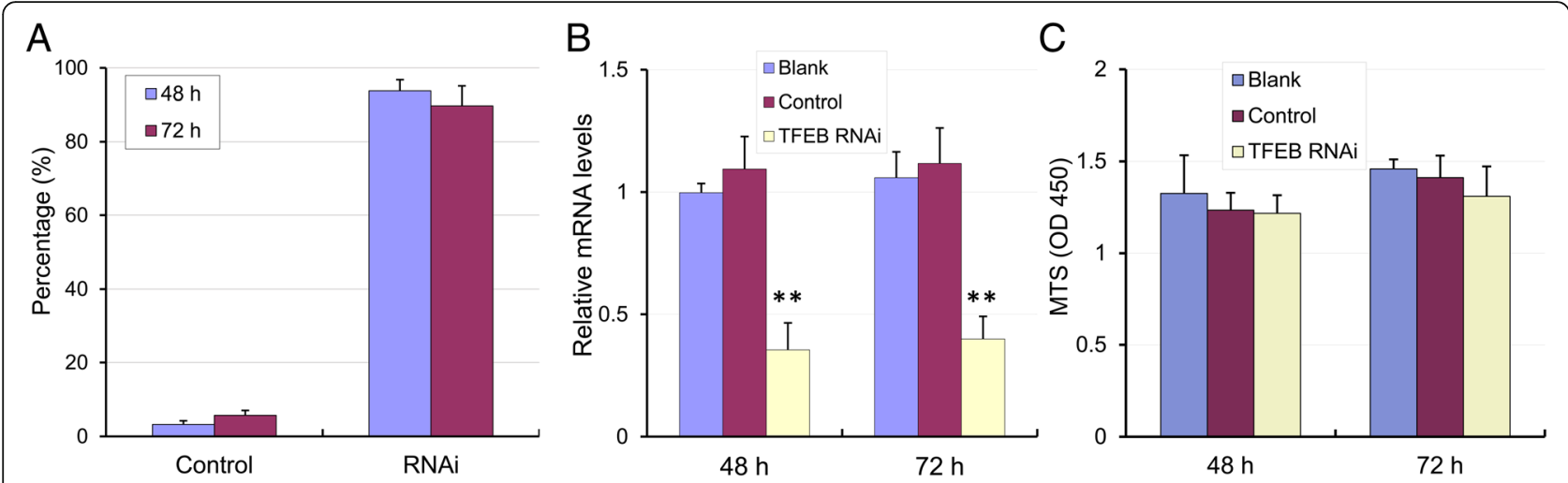

Fig. 5 Knock down of TFEB by shRNA lentiviral transduction in GC-1 cells. a The lentiviral infection efficiency was accessed by detection of the EGFP using flow cytometer analysis. After lentiviral transduction for $48 \mathrm{~h}$ and $72 \mathrm{~h}$, shRNA lentiviral expression efficiency estimated at $94 \pm 3 \%$ and $90 \pm 6 \%$, respectively $(n=4)$. b The relative mRNA levels of TFEB after lentiviral transduction for $48 \mathrm{~h}$ and $72 \mathrm{~h}$ were $0.35 \pm 0.11$ and $0.40 \pm 0.09$, comparing to the controls $\left(n=6,{ }^{* *} p<0.01\right)$. c Cell survival assay performed with MTS method, showed no significant difference after shRNA lentiviral infection for $48 \mathrm{~h}$ and $72 \mathrm{~h}(n=6)$

TFEB has been characterized as a master regulator of lysosomal biogenesis and autophagy that promotes the intracellular clearance or recycling of cellular components. Notably, autophagy and endocytosis can efficiently transport sets of transcription factors, adhesion molecules, or secreted factors, and therefore has been recently recognized as a major mechanism in the regulation of self-renewal and differentiation of stem cells as well as a regulator of cell migration [37-39]. Meanwhile, the maintenance of sustained spermatogenesis relies on the balance between spermatogonia self-renewal and differentiation. Previous studies have shown that the mechanistic target of rapamycin complex 1 (mTORC1) is the central regulator of this balance, while it also negatively regulated TFEB activity and restricted the induction of autophagy $[40,41]$. These results prompted us to evaluate the relationship between TFEB expression and spermatogenesis, during which several important processes, i.e. spermatogonia differentiation, germ cell migration, acrosome biogenesis and spermiation, seem related to lysosomal function and autophagy.

Interestingly, in mouse testicular development, the expression of TFEB was rapidly increased in the period from $7 \mathrm{dpp}$ to $14 \mathrm{dpp}$, during which spermatogonia underwent differentiation induced by RA in order to enter the prophase of the first meiotic division. Meanwhile, in the adult testis, the distribution of TFEB appears to be focused in the differentiating spermatogonia at stages VI to VIII of the seminiferous epithelial cycle, which coincides with the germ cell stages that are exposed to the highest levels of RA that promote spermatogonia differentiation, meiotic initiation and preL migrating across the BTB $[6,7]$. This seems to indicate that TFEB exhibits a potential association with RA signaling and may be involved in the differentiation of spermatogonia and the release of differentiating germ cells from the basal lamina to migrate across the seminiferous epithelium.

However, the expression pattern of TFEB in testis was insufficient to access the role of TFEB in spermatogonia. It is necessary to purify the spermatogonia and study the TFEB in spermatogonia in vitro. Notably, in 7 dpp testes, spermatogonia are undergoing differentiation but do not initiate meiosis. Thus, both undifferentiated and differentiating spermatogonia account for a large proportion in $7 \mathrm{dpp}$ testes, which is propitious to the isolation of spermatogonia without hindrance from excess spermatocyte and spermatid. Therefore, both undifferentiated and differentiating spermatogonia were isolated from $7 \mathrm{dpp}$ testes successfully by using magnetic-activated cell sorting and the isolated undifferentiated spermatogonia were further cultured with a feeder cell free method. Cell morphology, monitored for over 15 days, showed a unique single, paired and aligned cell arrangement, analogous to the A-single, A-paired and A-aligned spermatogonia in the testis. More importantly, these cultured, undifferentiated spermatogonia could undergo differentiation induced by RA treatment. Our analyses suggest an important model wherein the highly pure populations of spermatogonia can be isolated by magnetic activated cell sorting and used to generate enriched cultures of mouse undifferentiated spermatogonia or spermatogonial stem cells for use in in vitro culture studies on spermatogonial differentiation and meiotic initiation. Based on this method, we found that TFEB was mainly expressed in differentiating spermatogonia and potentially activated by RA signaling, consistent with its expression pattern in testicular sections. As previously described, RA is a novel inducer of spermatogonia differentiation and meiotic initiation $[6,7]$. RA 


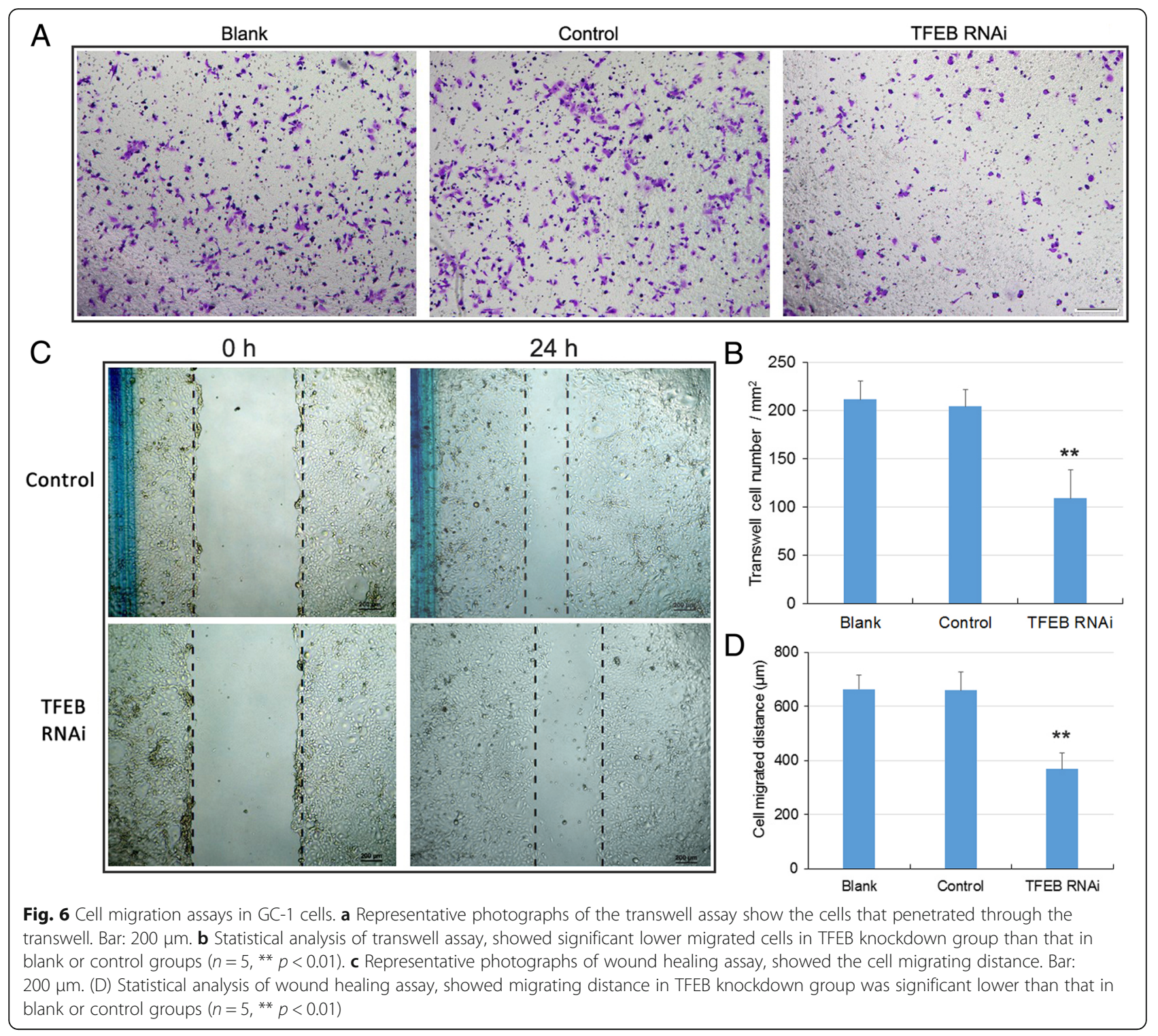

associated with retinoic acid receptors (RAR) activates the transcription of Stimulated by retinoic acid gene 8 (Stra8) and Rec8, as well as the kinase activity of PI3K/PDK1/ AKT/mTORC1 signaling pathways, which are required for translation of Kit, Sohlh1, and Sohlh2, and promote spermatogonial differentiation $[42,43]$. Therefore, we hypothesis that RA signaling might either promote TFEB transcription or regulate TFEB activity through kinase signaling pathways. The association of TFEB activation with RA signaling further highlights a potential role for TFEB in the regulation of certain process during spermatogonial differentiation and meiotic initiation.

Yet, less is known on the mechanisms regulating spermatogonial differentiation [44]. The processes leading from spermatogonial stem cells (SSC) to type B spermatogonia and preL have been seldom investigated.
Thus, it is important to better understand the molecular mechanisms involving in these processes. Our data provides a potential candidate responsible for spermatogonial differentiation induces by RA. Thus, this evidence prompted our research to focus on the TFEB function in spermatogonial differentiation. Until now, the biological relevance of TFEB to spermatogonia function or spermatogenesis has largely remained unexplored. Here, we attempted to determine the function of TFEB by RNA interference in cultured spermatogonia. Despite the reduction in TFEB mRNA expression levels, the anticipated decline in the levels of spermatogonial differentiating markers was not observed, which suggests other possible function of TFEB than directly induce spermatogonia differentiation should be taken into consideration. 
Therefore, considering that TFEB is a master regulator of lysosomal biogenesis and autophagy, we hypothesized that TFEB expression in differentiating spermatogonia may promote cell migration that is required for crossing the BTB. Recent studies have found that knockdown of TFEB by siRNA transfection has no effects on proliferation but diminishes migration abilities in several cancer cell lines $[28,29]$. Here, our study provides the first evidence for the role of TFEB in spermatogonial migration. Primary cultured spermatogonia were inadaptable to the classical cell migration assays because of the low cellular density and poor cell adhesion. In a previous study, Chen et al. performed a migration assay in immortalized spermatogonia, GC-1 cells [36]. Although it might lose some characteristics of normal spermatogonia, GC-1 cells are the most compatible cell line representing spermatogonia and are applied in many experiments to evaluate spermatogonia function in vitro [45-47]. Moreover, TFEB expressed in GC-1 cells also exhibited nucleus translocation following RA treatment (Additional file 1: Figure S3), which was consistent with that in primary cultured spermatogonia. This result indicated to the extent that GC-1 cells were appropriate for studying the effect of TFEB. Thus, in present study, GC-1 cells were also applied in cell migration experiments using the transwell and wound healing methods. The results confirmed that knockdown of TFEB led to an impairment in cell migration and indicated the potential effect of TFEB on spermatogonia migration, which are important and guided for the further study. Although the mechanisms by which the TFEB impacts migration is currently unknown, one possible explanation is that TFEB is involved in the degradation of matrix proteins, adhesion proteins and chemotactic receptors by activation of endocytosis, autophagy and lysosomal degradation.

On the other hand, the underlying mechanism for regulating migration of spermatogonia or spermatocytes is mostly unknown at present. Previous studies on germ cell transport or migration across the seminiferous epithelium, most notably the transport of preL across the BTB and the transport of elongating spermatids across the adluminal compartment, are focused on the adhesion protein complexes at the cell-cell interface [48-50]. It is generally accepted that cell junctions elaborated by Sertoli cells, namely both Sertoli-Sertoli cell and Sertoli-germ cell junctions, contribute to germ cells migration across the seminiferous epithelium. Emerging evidence has shown that junction dynamics at the Sertoli-Sertoli cell and Sertoli-germ cell interface are supported by the intriguingly coordinated cytoskeletons, microfilament- and microtubule-based cytoskeletons. However, apart from the effect of Sertoli cells, the molecular mechanisms in germ cell itself for its timely and efficient migration across the BTB remain virtually unknown. Limited evidences of the mechanisms in germ cell migration were found in studies on the homing or migration of pro-spermatogonia into the SSC niche. Earlier researches suggested that small $G$ protein Rac1 and transcription factor RHOX10 are critical regulators of spermatogonial stem cells (SSC) transmigrate through the BTB and migrate into niches during SSC homing $[51,52]$. Herein, our results indicate that TFEB as a transcriptional factor in spermatogonia has potential function in promoting spermatogonia migration, therefore provide a new candidate responsible for spermatogonia migration.

Whereas additional studies are required to understand precisely how stage-specific expression and activation of TFEB are regulated by RA signaling and how its downstream target genes impact on spermatogenesis, our immediate goal was to set the stage for assessing the role of TFEB in spermatogonia.

\section{Conclusions}

In conclusion, we demonstrated, for the first time, that TFEB expressed in spermatogonia in mouse testis and its expression was associated with spermatogonial differentiation and meiotic initiation. These data suggest that regionally distinct expression and activation of TFEB was strongly associated with RA signaling, and therefore may contribute to promote cell migration across the BTB and transport along the seminiferous epithelium.

\section{Additional file}

Additional file 1: Figure S1. Isolation and culture of spermatogonia from 7-day testes. (a-b) Enzymatic digestion of seminiferous tubules into fragments and single cells. (c) Testicular single cells were further performed differential attachment, showed the somatic cells present as triangle- or spindle-liked forms were attached to the plate, whereas the round unattached cells were spermatogonia. (d) Spermatogonia purified by magnetic-activated cell sorting. (e) Culture of the somatic cells isolated by differential attachment, containing Sertoli cells and myoid cells. (f) Culture of the Thy 1 positive spermatogonia. Bar: $200 \mu \mathrm{m}$ in (a), (b); $100 \mu \mathrm{m}$ in (c)-(e); $50 \mu \mathrm{m}$ in (f). Figure S2. Immunofluorescent staining of GFRA1 in cultured spermatogonia. The result showed most cells were positive for GFRA1, suggesting high purity of undifferentiated spermatogonia. Bars: $20 \mu \mathrm{m}$. Figure S3. Immunofluorescent staining of TFEB in GC-1 cells. The result showed that RA treatment induced TFEB nucleus translocation. Bars: $20 \mu \mathrm{m}$. Table S1. Oligonucleotide primer sequences used for qRTPCR. (DOCX 3401 kb)

\section{Abbreviations}

ANOVA: One-way analysis of variance; BSA: Bovine serum albumin; BTB: Blood-testis barrier; dpp: Days post-partum; EGFP: Enhanced green fluorescence protein; FBS: Fetal bovine serum; GFRA1: GDNF family receptor alpha 1; H\&E: Hematoxylin and eosin; IHC: Immunohistochemistry; mTORC1: Mechanistic target of rapamycin complex 1; preL: Preleptotene spermatocytes; PVDF: Polyvinylidene difluoride; RA: Retinoic acid; RTPCR: Reverse transcription-polymerase chain reaction; shRNA: Short hairpin RNA; TFEB: Transcriptional factor EB 


\section{Acknowledgements}

The authors thank Ms. Rong Fu (Core Facility of Basic Medical Sciences) for her technical assistance in flow cytometry assay. The authors are grateful to Dr. Michael Van for critically proofreading the manuscript.

\section{Funding}

This research project was supported by grants from the National Natural Science Foundation of China (No. 81701503 and 81471497) and the Shanghai Municipal Commission of Health and Family Planning (No. 20134115).

\section{Availability of data and materials}

All data generated or analyzed during this study are included in this published article and its supplementary information files.

\section{Authors' contributions}

YL conducted and performed experiments, analyzed data, and prepared the manuscript. YH performed histological analysis. LW performed part of molecular experiments. CX supervised the project and finally approved the manuscript. All authors read and approved the final manuscript.

\section{Ethics approval}

All animal care and experimental protocols used in this study were approved by the institutional animal care and the ethic committee of Shanghai Jiao Tong University School of Medicine (NO. A2015-034).

\section{Consent for publication}

Not applicable.

\section{Competing interests}

The authors declare that they have no competing interests.

\section{Publisher's Note}

Springer Nature remains neutral with regard to jurisdictional claims in published maps and institutional affiliations.

\section{Author details}

'Department of Histology, Embryology, Genetics and Developmental Biology, Shanghai Jiao Tong University School of Medicine, Shanghai 200025, China. ${ }^{2}$ Shanghai Key Laboratory for Reproductive Medicine, Shanghai 200025 China.

Received: 29 August 2018 Accepted: 17 October 2018 Published online: 25 October 2018

\section{References}

1. Nishimura H, L'Hernault SW. Spermatogenesis. Curr Biol. 2017;27:988-94.

2. Mecklenburg JM, Hermann BP. Mechanisms Regulating Spermatogonial Differentiation. In: Piprek R. (eds) Molecular Mechanisms of Cell Differentiation in Gonad Development. Results and Problems in Cell Differentiation. Springer, Cham; 2016. p253-287.

3. Song HW, Wilkinson MF. Transcriptional control of spermatogonia maintenance and differentiation. Semin Cell Dev Biol. 2014;30:14-26.

4. Li L, Gao Y, Chen H, Jesus T, Tang E, Li N, Lian Q, Ge RS, Cheng CY. Cell polarity, cell adhesion, and spermatogenesis: role of cytoskeletons. F1000Res. 2017:6:1565.

5. Xiao X, Mruk DD, Wong CKC, Cheng CY. Germ cell transport across the seminiferous epithelium during spermatogenesis. Physiology. 2014;29: 286-98.

6. Busada JT, Geyer CB. The role of retinoic acid (RA) in Spermatogonial differentiation. Biol Reprod. 2016;94:10.

7. Griswold MD. Spermatogenesis: the commitment to meiosis. Physiol Rev. 2016;96:1-17.

8. Mruk DD, Cheng CY. Sertoli-Sertoli and Sertoli-germ cell interactions and their significance in germ cell movement in the seminiferous epithelium during spermatogenesis. Endocr Rev. 2004;25:747-806.

9. Wen Q, Tang El, Xiao X, Gao Y, Chu DS, Mruk DD, Silvestrini B, Cheng CY. Transport of germ cells across the seminiferous epithelium during spermatogenesis-the involvement of both actin- and microtubule-based cytoskeletons. Tissue Barriers. 2016;4:e1265042.
10. Coly PM, Gandolfo P, Castel H, Morin F. The autophagy machinery: a new player in chemotactic cell migration. Front Neurosci. 2017;11:78.

11. Kawano S, Torisu T, Esaki M, Torisu K, Matsuno Y, Kitazono T. Autophagy promotes degradation of internalized collagen and regulates distribution of focal adhesions to suppress cell adhesion. Biol Open. 2017;6:1644-53.

12. Kenific CM, Wittmann T, Debnath J. Autophagy in adhesion and migration. J Cell Sci. 2016;129:3685-93.

13. Maritzen T, Schachtner H, Legler DF. On the move: endocytic trafficking in cell migration. Cell Mol Life Sci. 2015;72:2119-34.

14. Paul NR, Jacquemet G, Caswell PT. Endocytic trafficking of Integrins in cell migration. Curr Biol. 2015;25:1092-105.

15. Schmid SL. Reciprocal regulation of signaling and endocytosis: implications for the evolving cancer cell. J Cell Biol. 2017;216:2623-32.

16. Pastore N, Brady OA, Diab HI, Martina JA, Sun L, Huynh T, Lim JA, Zare H, Raben N, Ballabio A, Puertollano R. TFEB and TFE3 cooperate in the regulation of the innate immune response in activated macrophages. Autophagy. 2016:12:1240-58.

17. Sardiello M, Palmieri M, di Ronza A, Medina DL, Valenza M, Gennarino VA, D Malta C, Donaudy F, Embrione V, Polishchuk RS, Banfi S, Parenti G, Cattaneo E, Ballabio A. A gene network regulating lysosomal biogenesis and function. Science. 2009:325:473-7.

18. Settembre C, Di Malta C, Polito VA, Garcia Arencibia M, Vetrini F, Erdin S, Erdin SU, Huynh T, Medina D, Colella P, et al. TFEB links autophagy to lysosomal biogenesis. Science. 2011;332:1429-33.

19. Napolitano G, Ballabio A. TFEB at a glance. J Cell Sci. 2016;129:2475-81.

20. Peña-Llopis S, Brugarolas J. TFEB, a novel mTORC1 effector implicated in lysosome biogenesis, endocytosis and autophagy. Cell Cycle. 2011;10:3987-8.

21. Song W, Wang F, Savini M, Ake A, di Ronza A, Sardiello M, Segatori L. TFEB regulates lysosomal proteostasis. Hum Mol Genet. 2013;22:1994-2009.

22. Martina JA, Chen Y, Gucek M, Puertollano R. MTORC1 functions as a transcriptional regulator of autophagy by preventing nuclear transport of TFEB. Autophagy. 2012:8:903-14.

23. Medina DL, Di Paola S, Peluso I, Armani A, De Stefani D, Venditti R, Montefusco S, Scotto-Rosato A, Prezioso C, Forrester A, et al. Lysosomal calcium signalling regulates autophagy through calcineurin and TFEB. Nat Cell Biol. 2015:17:288-99.

24. Puertollano R, Ferguson SM, Brugarolas J, Ballabio A. The complex relationship between TFEB transcription factor phosphorylation and subcellular localization. EMBO J. 2018;37:e98804.

25. Settembre C, De Cegli R, Mansueto G, Saha PK, Vetrini F, Visvikis O, Huynh T, Carissimo A, Palmer D, Klisch TJ, et al. TFEB controls cellular lipid metabolism through a starvation-induced autoregulatory loop. Nat Cell Biol. 2013;15:647-58.

26. Settembre C, Ballabio A. Lysosome: regulator of lipid degradation pathways. Trends Cell Biol. 2014:24:743-50.

27. Tong $Y$, Song F. Intracellular calcium signaling regulates autophagy via calcineurin-mediated TFEB dephosphorylation. Autophagy. 2015;11: 1192-5.

28. Giatromanolaki A, Kalamida D, Sivridis E, Karagounis IV, Gatter KC, Harris AL, Koukourakis MI. Increased expression of transcription factor EB (TFEB) is associated with autophagy, migratory phenotype and poor prognosis in non-small cell lung cancer. Lung Cancer. 2015;90:98-105.

29. Sakamoto H, Yamashita K, Okamoto K, Kadowaki T, Sakai E, Umeda M, Tsukuba T. Transcription factor EB influences invasion and migration in oral squamous cell carcinomas. Oral Dis. 2018;24:741-8.

30. He Z, Kokkinaki M, Jiang J, Dobrinski I, Dym M. Isolation, characterization, and culture of human spermatogonia. Biol Reprod. 2010;82:363-72.

31. Liu Y, Chen G, Lu L, Sun H, Guo Q, Xue K, Fan Y, Ding Z. RNASET2 in human spermatozoa and seminal plasma: a novel relevant indicator for asthenozoospermia. Andrology. 2013;1:75-84.

32. Holembowski L, Kramer D, Riedel D, Sordella R, Nemajerova A, Dobbelstein M, Moll UM. TAp73 is essential for germ cell adhesion and maturation in testis. J Cell Biol. 2014;204:1173-90

33. Sato Y, Yoshida K, Nozawa S, Yoshiike M, Arai M, Otoi T, Iwamoto T. Establishment of adult mouse Sertoli cell lines by using the starvation method. Reproduction. 2013;145:505-16.

34. Huszar JM, Payne CJ. MicroRNA 146 (Mir146) modulates spermatogonial differentiation by retinoic acid in mice. Biol Reprod. 2013:88:15.

35. Choi NY, Park YS, Ryu JS, Lee HJ, Araúzo-Bravo MJ, Ko K, Han DW, Schöler HR, Ko K. A novel feeder-free culture system for expansion of mouse spermatogonial stem cells. Mol Cells. 2014;37:473-9. 
36. Chen H, Fok KL, Yu S, Jiang J, Chen Z, Gui Y, Cai Z, Chan HC. CD147 is required for matrix metalloproteinases-2 production and germ cell migration during spermatogenesis. Mol Hum Reprod. 2011;17:405-14.

37. Boya $\mathrm{P}$, Codogno $\mathrm{P}$, Rodriguez-Muela N. Autophagy in stem cells: repair, remodelling and metabolic reprogramming. Development. 2018;145:146506.

38. Brunt L, Scholpp S. The function of endocytosis in Wnt signaling. Cell Mol Life Sci. 2018;75:785-95.

39. Chen $X$, He Y, Lu F. Autophagy in stem cell biology: a perspective on stem cell self-renewal and differentiation. Stem Cells Int. 2018;2018:9131397.

40. Busada JT, Niedenberger BA, Velte EK, Keiper BD, Geyer CB. Mammalian target of rapamycin complex 1 (mTORC1) is required for mouse spermatogonial differentiation in vivo. Dev Biol. 2015;407:90-102.

41. Hobbs RM, Seandel M, Falciatori I, Rafii S, Pandolfi PP. Plzf regulates germline progenitor self-renewal by opposing mTORC1. Cell. 2010;142: 468-79.

42. Endo T, Freinkman E, de Rooij DG, Page DC. Periodic production of retinoic acid by meiotic and somatic cells coordinates four transitions in mouse spermatogenesis. Proc Natl Acad Sci U S A. 2017;114:10132-10141.

43. Chen SR, Liu YX. Regulation of spermatogonial stem cell self-renewal and spermatocyte meiosis by Sertoli cell signaling. Reproduction. 2015;149:159-67.

44. Manku G, Culty M. Mammalian gonocyte and spermatogonia differentiation: recent advances and remaining challenges. Reproduction. 2015;149:139-57.

45. Li C, Chen S, Li H, Chen L, Zhao Y, Jiang Y, Liu Z, Liu Y, Gao S, Wang F, et al. MicroRNA-16 modulates melatonin-induced cell growth in the mousederived Spermatogonia cell line GC-1 spg cells by targeting Cond1. Biol Reprod. 2016:95:57.

46. Santillo A, Falvo S, Chieffi P, Di Fiore MM, Senese R, Chieffi Baccari G. Daspartate induces proliferative pathways in Spermatogonial GC-1 cells. J Cell Physiol. 2016;231:490-5.

47. Zhao L, Zhu Z, Yao C, Huang Y, Zhi E, Chen H, Tian R, Li P, Yuan Q, Xue Y, et al. VEGFCNEGFR3 signaling regulates mouse Spermatogonial cell proliferation via the activation of AKT/MAPK and cyclin D1 pathway and mediates the apoptosis by affecting caspase 3/9 and Bcl-2. Cell Cycle. 2018; 17:225-39.

48. Zhang J, Wong CH, Xia W, Mruk DD, Lee NP, Lee WM, Cheng CY. Regulation of Sertoli-germ cell adherens junction dynamics via changes in proteinprotein interactions of the $\mathrm{N}$-cadherin-beta-catenin protein complex which are possibly mediated by c-Src and myotubularin-related protein 2: an in vivo study using an androgen suppression model. Endocrinology. 2005;146: 1268-84.

49. Hannigan MM, Zagore LL, Licatalosi DD. Ptbp2 Controls an Alternative Splicing Network Required for Cell Communication during Spermatogenesis. Cell Rep. 2017;19:2598-2612.

50. Holembowski L, Kramer D, Riedel D, Sordella R, Nemajerova A, Dobbelstein $M$, Moll UM. TAp73 is essential for germ cell adhesion and maturation in testis. J Cell Biol. 2014;204:1173-1190.

51. Takashima S, Kanatsu-Shinohara M, Tanaka T, Takehashi M, Morimoto H, Shinohara T. Rac mediates mouse spermatogonial stem cell homing to germline niches by regulating transmigration through the blood-testis barrier. Cell Stem Cell. 2011:9:463-75.

52. Song HW, Bettegowda A, Lake BB, Zhao AH, Skarbrevik D, Babajanian E, Sukhwani M, Shum EY, Phan MH, Plank TM, et al. The Homeobox transcription factor RHOX10 drives mouse Spermatogonial stem cell establishment. Cell Rep. 2016;17:149-64.

\section{Ready to submit your research? Choose BMC and benefit from:}

- fast, convenient online submission

- thorough peer review by experienced researchers in your field

- rapid publication on acceptance

- support for research data, including large and complex data types

- gold Open Access which fosters wider collaboration and increased citations

- maximum visibility for your research: over $100 \mathrm{M}$ website views per year

At BMC, research is always in progress.

Learn more biomedcentral.com/submissions 